\title{
3D scanning particle tracking velocimetry
}

\section{Journal Article}

\section{Author(s):}

Hoyer, Klaus; Holzner, Markus; Lüthi, Beat; Guala, Michele; Liberzon, Alex; Kinzelbach, Wolfgang

Publication date:

2005-11

\section{Permanent link:}

https://doi.org/10.3929/ethz-b-000026482

\section{Rights / license:}

In Copyright - Non-Commercial Use Permitted

\section{Originally published in:}

Experiments in Fluids 39(5), https://doi.org/10.1007/s00348-005-0031-7 


\author{
Klaus Hoyer • Markus Holzner • Beat Lüthi \\ Michele Guala $\cdot$ Alexander Liberzon \\ Wolfgang Kinzelbach
}

\title{
3D scanning particle tracking velocimetry
}

Received: 10 March 2005/ Revised: 1 July 2005/ Accepted: 19 July 2005 / Published online: 25 August 2005

(C) Springer-Verlag 2005

\begin{abstract}
In this article, we present an experimental setup and data processing schemes for 3D scanning particle tracking velocimetry (SPTV), which expands on the classical 3D particle tracking velocimetry (PTV) through changes in the illumination, image acquisition and analysis. 3D PTV is a flexible flow measurement technique based on the processing of stereoscopic images of flow tracer particles. The technique allows obtaining Lagrangian flow information directly from measured 3D trajectories of individual particles. While for a classical PTV the entire region of interest is simultaneously illuminated and recorded, in SPTV the flow field is recorded by sequential tomographic high-speed imaging of the region of interest. The advantage of the presented method is a considerable increase in maximum feasible seeding density. Results are shown for an experiment in homogenous turbulence and compared with PTV. SPTV yielded an average 3,500 tracked particles per time step, which implies a significant enhancement of the spatial resolution for Lagrangian flow measurements.
\end{abstract}

Keywords Scanning $\cdot$ Tracking $\cdot$ Lagrangian

\section{Introduction}

Particle tracking velocimetry (PTV) has been established in an automated form as a valuable tool for Lagrangian flow measurements in the 1980s. Chang

K. Hoyer $(\bowtie) \cdot$ M. Holzner $\cdot$ M. Guala $\cdot$ A. Liberzon

W. Kinzelbach

Institut für Hydromechanik und Wasserwirtschaft,

Swiss Federal Institut of Technology Zurich,

ETH Hönggerberg, 8093 Zürich, Switzerland

E-mail: hoyer@ihw.baug.ethz.ch

B. Lüthi

Risø National Laboratory, Frederiksborgvej 399,

P.O. Box 49, 4000 Roskilde, Denmark and Tatterson (1983) introduced a stereoscopic 3D PTV system, further developed by Racca and Dewey (1988). Nishino et al. (1988) presented a 3D PTV system that allowed arbitrary viewing directions and yielded, on average, about 160 velocity vectors in a measurement of an unsteady laminar Couette flow. Later, Kasagi et al. (1991) applied the system to a turbulent channel flow where they presented flow measurements and flow statistics from about 440 instantaneous velocity vectors. Papantoniou et al. (1990) succeeded in tracking about 700 particles simultaneously using a three-camera system. Thereafter, Maas et al. (1993) were able to increase the number of simultaneous velocity vectors to about 1,300 per time step in a 3D turbulent channel flow. The method has been developed, tested and validated during the course of several flow studies (most recently in Lüthi et al. 2005, see also Dracos 1996; Maas 1992; Willneff 2003). However, the number of $O\left(10^{3}\right)$ velocity vectors still represents the achievable limit when using a classical PTV system consisting of four standard CCD cameras. This limit is imposed mainly by ambiguities of particle positions arising from particle image overlap on the imager chip of the camera. Ambiguities can be tolerated to a certain extent; they become, however, prohibitive with further increase of the seeding density.

For classical 3D PTV, a multi-camera system is needed to image an observation volume with an aspect ratio of $O(1)$, i.e. the extent of the observation volume in the direction of the optical axes of the cameras has a dimension comparable to the width and height. The three velocity components are obtained by identifying and tracking the position of the particles as they move through the observation volume. Lüthi et al. (2005) measured the full set of velocity derivatives in a Lagrangian way in quasi-homogeneous turbulence. As pointed out by the authors in Lüthi et al. (2005), for a better accuracy of the velocity derivatives, both longparticle trajectories and high spatial resolution are desirable. As mentioned above, with increasing seeding density, ambiguities in individual particle recognition 
occur due to the large third dimension of the observation volume. This results in noisy and incomplete data and thus hinders the tracking of particles over many time steps. Long trajectories could be determined so far only if the probability of ambiguities is reduced by lowering the seeding density, however, concurrently reducing the spatial resolution.

In scanning PTV, this problem is overcome by subdividing the observation volume into a series of slices, each much thinner along the optical axes of the cameras. This allows for an increase in seeding density accordingly, since the saturation of particle images will be limited to a single slice only. The concept of a scanning light sheet is not new in whole-field velocity measurements. Whole-field velocity measurements were reported by Merkel et al. (1998) and $\mathrm{Su}$ and Dahm (1996a, b) using tomographic laser-induced florescence (LIF) and also by Brücker (1997), who used a scanning laser beam which provided overlapping illumination to correlate the particle images not only in the $2 \mathrm{D}$ in plane coordinates $x_{1}$ and $x_{2}$, but also to obtain a correlation in the out of plane direction $x_{3}$, to measure velocity components in the third dimension. The necessary considerable overlap of the light sheets for the correlation technique can also be avoided when using stereoscopic scanning PIV (Brücker 1996a) or colorcoded PIV (Brücker 1996b). A combination of 2D correlation with subsequent 2D tracking of individual particles allowed Guezennec at al. (1994) to increase the in-plane spatial resolution of their stereoscopic scanning PIV images considerably compared to the use of correlation only. Using an adaptive Gaussian filter, they interpolated the velocities onto a regular grid for each of their stored images and thus obtained an inplane high-resolution velocity vector map of the three velocity components combining two camera projections. Both tomographic LIF and tomographic PIV require fairly thin illuminated layers. In LIF this is especially true because of the necessity to have nonblurred images with a resolution similar in the scanning direction as in the laser plane itself. Therefore with tomographic LIF one obtains volumetric data of the scalar concentration field with very high spatial resolution. However, the scalar gradient field has to be mixed and folded sufficiently to have measurable scalar gradients down to the smallest eddy scales, i.e. the Kolmogorov dissipation scale. For scanning PIV, both $2 \mathrm{D}$ and stereo, the light sheet thickness is determined by the fairly high seeding density of tracer particles together with the desired small interrogation volume. Additionally, in PIV, the uncertainty of the position of the velocity vector is determined by the light sheet thickness and also for this reason one needs to keep the sheet as thin as possible. These restrictions require the experimenter to carefully compromise on the variation of possible parameters. These are mainly related to light intensity, sheet thickness, particle size, seeding density, overall achievable volume scan rate with a given camera, measurement location and, especially in tomographic imaging, the restriction of the depth of field. In the presented scanning particle tracking velocimetry (SPTV) technique, the accuracy of an individual particle position is mainly determined by the viewing angles, camera resolution and calibration quality, while the thickness of the light sheet plays no direct role. Therefore, in SPTV the restriction of the light sheet thickness is nowhere as critical as required by LIF and PIV. In fact, the sheet thickness can vary together with the seeding density such that we are close to the established limit of $O\left(10^{3}\right)$ particles per slice. For a fixed-camera frame rate, one can therefore vary the volume scan rate together with the number and thickness of the image planes and the particle seeding density to the optimum values for the experiment. In other words, depending on the experimental conditions, one can scan with a high seeding density and a larger number of thin slices using a slower volume scan rate or one can scan with a lower seeding density, a smaller number of thicker slices and a higher volume scan rate.

Tomographic LIF was also performed by Sakakibara and Adrian (1999) in the measurement of a 3D temperature field using a mixture of Rhodamine B and Rhodamine 110 fluorescent dies. The temperature field was determined from the previously calibrated temperature-dependent ratio of the two dies' fluorescent intensities, which were separated using a color beam splitter. Although the primary goal was the measurement of the temperature field, the temperature itself could be used as a flow marker to derive the velocity field. Recently, Hori and Sakakibara (2004) published results from a scanning PIV system measuring a $100 \mathrm{~mm}^{3}$ observation volume of a round jet of Reynolds number 1,000 . They obtained velocity vectors on a grid spaced in $x_{1}, x_{2}$ and $x_{3}$ directions of $2.5 \mathrm{~mm} \times$ $3.5 \mathrm{~mm} \times 2 \mathrm{~mm}$, respectively. This is equivalent to measuring on a grid of $40 \times 28 \times 50$ mesh points. The volume scan rate of $3 \mathrm{~Hz}$ achieved in their experiment, however, restricts the application considerably. When one compares the different techniques, all have advantages in some respect and compromise elsewhere, mainly to benefit either the spatial or temporal resolution of the field of view. PTV/SPTV, however, is the only method that directly yields Lagrangian flow information. This is of utmost importance for the study of mixing and transport phenomena and for the investigation of turbulence characteristics in general.

The main processing steps in particle tracking are (1) in situ calibration, (2) stereoscopic matching, (3) tracking of individual particles and (4) post-processing of trajectories.

With the exception of the in situ calibration, all processing and post-processing steps require modifications for SPTV. The necessary modifications on the image acquisition side are discussed in Sect. 2 and the processing modifications in Sect. 3. Results for an experiment in homogenous turbulence are presented in Sect. 4 and conclusions are drawn in Sect. 5. 


\section{Experimental apparatus and setup}

\subsection{Hardware}

Scanning particle tracking velocimetry uses images that are obtained by a high-speed camera system recording scattered light from particles illuminated by a scanning laser light sheet. To obtain a time resolution comparable to the classical PTV technique, the SPTV system has to scan through a whole volume in the same time as the classical PTV system needs to record a single frame. Therefore the camera recording rate has to be faster, proportionally to the number of slices in the observation volume. The camera frame rate $f_{\mathrm{c}}$ and the volume scan rate $f_{\mathrm{v}}$ are therefore linked in the following form through the number of slices $n_{\mathrm{s}}$ :

$f_{\mathrm{c}} \geq n_{\mathrm{s}} f_{\mathrm{v}}$.

The additional degree of freedom that one has in selecting the number of slices allows for fine-tuning of the seeding density to the necessary spatial and time scales for resolving the flow. This means that, for a given camera frame rate, one has the choice to either scan with few slices and a fast volume scan rate or with more slices and smaller volume scan rate, thereby increasing the spatial resolution by reducing the time resolution or vice versa.

An important parameter that has to be taken into account for this decision is the so-called "tracking parameter". The tracking procedure must establish correct links of individual particles between successive volume scans. A general criterion for the trackability of particles is given by the tracking parameter $p$, defined as the ratio between the mean displacement $d_{0}$ to the mean inter-particle distance $d_{p}$ (see Dracos 1996). For a small ratio, i.e. $d_{0}<d_{p}$, tracking is trivial because in most cases the nearest neighbor in the following time step is the right choice. For a high ratio, i.e. $d_{0}>d_{p}$, tracking becomes difficult and if the temporal resolution cannot be improved by hardware, the seeding of particles has to be decreased.

Our light source for illumination is a continuous $20 \mathrm{~W}$ argon ion laser. A beam expander comprising two cylindrical lenses spreads the beam to a sheet. A spherical lens of focal length $f=1 \mathrm{~m}$ is used to adjust the thickness of the light sheet. Following this set of lenses, the vertical light sheet passes through an octagonal Plexiglas cylinder (index of refraction $n_{2}=1.49$ ). This cylinder can rotate at a set speed around its axis while the laser light sheet passes through the parallel facing planes of the cylinder. Compared to the discrete step scan of mirror setups, with this setup the light sheet is scanned in a continuous way. It is flexible since the control of the light sheet thickness is achieved through the camera timing. The maximum sweep of the laser sheet, however, is tied to the geometry and requires a different octagon according to the desired region of interest. The cylinder axis is mounted vertically and aligned carefully with the incoming light sheet. Through its rotation, the angle of incidence of the incoming laser light sheet varies as $\dot{\phi}_{0}=2 \pi \mathrm{rad} / \mathrm{s}$ and is limited by the octagonal shape of the cylinder to $\left(-(\pi / 8)<\phi_{0}<(\pi / 8)\right)$. Each time the limiting angle is passed, the incident laser light sheet enters through the next face of the octagon starting at a new angle shifted by $-\pi / 4$. For each eighth of a rotation of this cylinder, the exiting light sheet shifts parallel by an amount of approximately $-10 \mathrm{~mm}<x_{3}<10 \mathrm{~mm}\left(x_{3}\right.$ is the coordinate parallel to the scanning direction and to the symmetry axis of the camera arrangement, respectively; see Fig. 1). This parallel shift depends non-linearly on the rotation angle and can be calculated using Snell's Law as follows:
Fig. 1 A schematic of the experimental setup. The laser beam is expanded to a light sheet and scanned through the observation volume using an eight-face prism

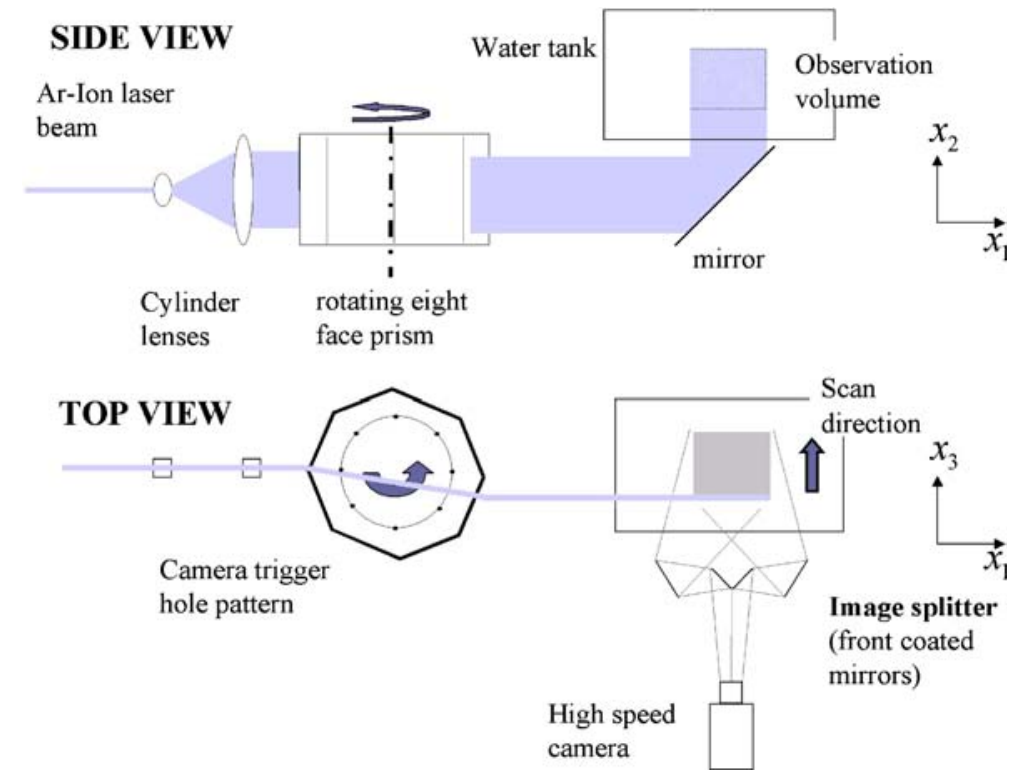


$x_{3}=h \sin \theta\left[1-\frac{\cos \theta}{\sqrt{\left(\frac{n_{1}}{n_{2}}\right)^{2}-(\sin \theta)^{2}}}\right]$,

where $h=85 \mathrm{~mm}$ is the inner circle diameter, $n_{1}=1.0$ is the refractive index of air, $n_{2}=1.49$ is the refractive index of the cylinder material and $\theta$ is the angle of incidence. Snell's Law is used in the presented technique for the determination of the slice position during data processing.

The high-speed camera used for the experiment is a Photron Ultima APX capable of achieving a maximum frame rate of $2 \mathrm{kHz}$ at full resolution $(1,024 \times 1,024$ pixels). With a built-in memory of $2 \mathrm{~GB}$, the system is able to record a time series of 2,000 images. We used a Nikor Micro $60 \mathrm{~mm}$ objective with an aperture set to $f 16$, which ensured good focus over the entire scanned depth of $20 \mathrm{~mm}$. The classical PTV system uses four synchronized cameras (Lüthi et al. 2005; Maas 1992) to enable robust stereoscopic matching within the established seeding limit, whereas in SPTV one single camera is used in combination with a four-way image splitter to mimic the classical four-camera setup. This four-way splitter consists of a set of four fixed primary beveled mirrors assembled on a regular pyramid and four secondary mirrors, which are mounted on regular pan/tilt fixtures to individually adjust the field of view for each viewing direction. All are front-coated mirrors. Through the projection of four viewing directions on a single camera chip, one has the advantage that exact synchronization of the cameras, which is critical for the stereoscopic matching and estimation of the particle positions, is automatically achieved. Through the four-way splitter a single camera image of $1,024 \times 1,024$ pixels resolution is split into four images, each of $512 \times 512$ pixels resolution, which is about the resolution of one CCD camera in the classical PTV system. As mentioned above, the minimum frame rate of the camera is determined by the revolution speed of the prism multiplied by the number of slices per volume scan. For the data presented, each volume scan consists of ten illuminated light sheets. With the revolution frequency of the octagonal cylinder set to $6.25 \mathrm{~Hz}$, we achieve a volume scan rate of $50 \mathrm{~Hz}$, which results in a minimum camera frame rate of $500 \mathrm{~Hz}$. With this setup we are able to record the observation volume 200 times during $4 \mathrm{~s}$. The rotating cylinder and the camera are synchronized by an external timing signal. This signal is generated by an infrared diode aligned over an eight-hole pattern, which is fixed and aligned with the corners of the rotating cylinder. Every time a cylinder corner crosses the light sheet, the camera records ten frames at a fixed rate of $500 \mathrm{~Hz}$.

The exposure time is set to the inverse of the frame rate, therefore the camera always integrates over the entire path of the moving light sheet. As mentioned above, the thickness of one single light sheet, $d_{\mathrm{s}}$, depends on the length of the scanning path during the exposure and on the 'static' light sheet thickness, which can be adjusted with a long focal length lens. Depending on these parameters, one achieves an illumination overlap region of variable size in which particles are exposed twice in successive scans. Although we use a continuous wave laser for the illumination and integrate the frame exposure over the inverse of the frame rate, we do not observe streaked particle images in this experiment. The time that a particle is illuminated by the incident laser light sheet during one scan depends on the static laser sheet thickness and the relative normal velocity of the particle with respect to the scan velocity. A particle that moves with the sheet (i.e. along the positive $x_{3}$-axis) has a smaller relative normal velocity and therefore is illuminated for a longer time than a particle that moves in the opposite direction. Since the flow velocities are considerably smaller than the scan velocity $v_{\mathrm{s}}$, this effect is very small. With a scan velocity $v_{\mathrm{s}} \approx 1 \mathrm{~m} / \mathrm{s}$ and the static light sheet thickness of about $1 \mathrm{~mm}$ we expect a particle illumination time of roughly $1 \mathrm{~ms}$ and the travel path of a particle during this time of about $10 \mu \mathrm{m}$. The travel path during the exposure therefore is smaller than the diameter of the particles. The highest resolvable frequency of the position signals in the flow is determined by the Nyquist criterion given a sample rate equal to the volume scan rate. With a volume scan rate of $50 \mathrm{~Hz}$ the Nyquist cutoff frequency lies at $25 \mathrm{~Hz}$. This cutoff is considerably higher than the highest expected frequency of the position signal of $4 \mathrm{~Hz}$ estimated from the Kolmogorov time scale $\left(\tau_{\eta}=0.25 \mathrm{~s}\right)$ in our flow.

\subsection{Flow}

Our flow apparatus is the same as used in Liberzon et al. (2005). The goal was to reproduce the same turbulent flow field as in Liberzon et al. 2005) and Lüthi et al. (2005), for a comparison to the classical PTV system. The forced flow domain is a rectangular box, $120 \times 120 \times 140 \mathrm{~mm}^{3}$. The flow is forced mechanically from two sides by two sets of four rotating disks. The disks have artificial roughness elements and are driven by a closed loop controlled servo motor. The motor is installed on top of the forcing unit and drives the counter-rotating disks through a fixed gear chain, where all disks rotate at the same rate according to the scheme shown in Fig. 2. The actual observation volume of approximately $20 \times 20 \times 20 \mathrm{~mm}^{3}$ was centered with respect to the forced flow domain, mid-way between the disks. The presented data were recorded with a disk rotational speed of $40 \mathrm{rpm}$. During the $4 \mathrm{~s}$ of the experiment the mean flow over the entire observation volume showed negligible mean velocity. The fluctuating rms velocity within the observation volume, $\sqrt{\bar{u}^{2}}$, is of the order $O\left(0.01 \mathrm{~m} \mathrm{~s}^{-1}\right)$. The characteristic properties of the flow are given in Table 1.

The flow is seeded with neutrally buoyant $40 \mu \mathrm{m}$ polystyrene particles $\left(\rho=1.02 \mathrm{~g} / \mathrm{cm}^{3}\right)$ with a number density of about 600 particles per $\mathrm{cm}^{3}$. This corresponds to an average inter-particle distance of $1.2 \mathrm{~mm}$. It is well 
Fig. 2 A servo-motor (not shown), installed on the top of the forcing unit, drives eight counter-rotating disks, four on each side rotating according to the scheme. The flow volume is located at the center of the forcing unit, mid-way between the disks fhb2.eps

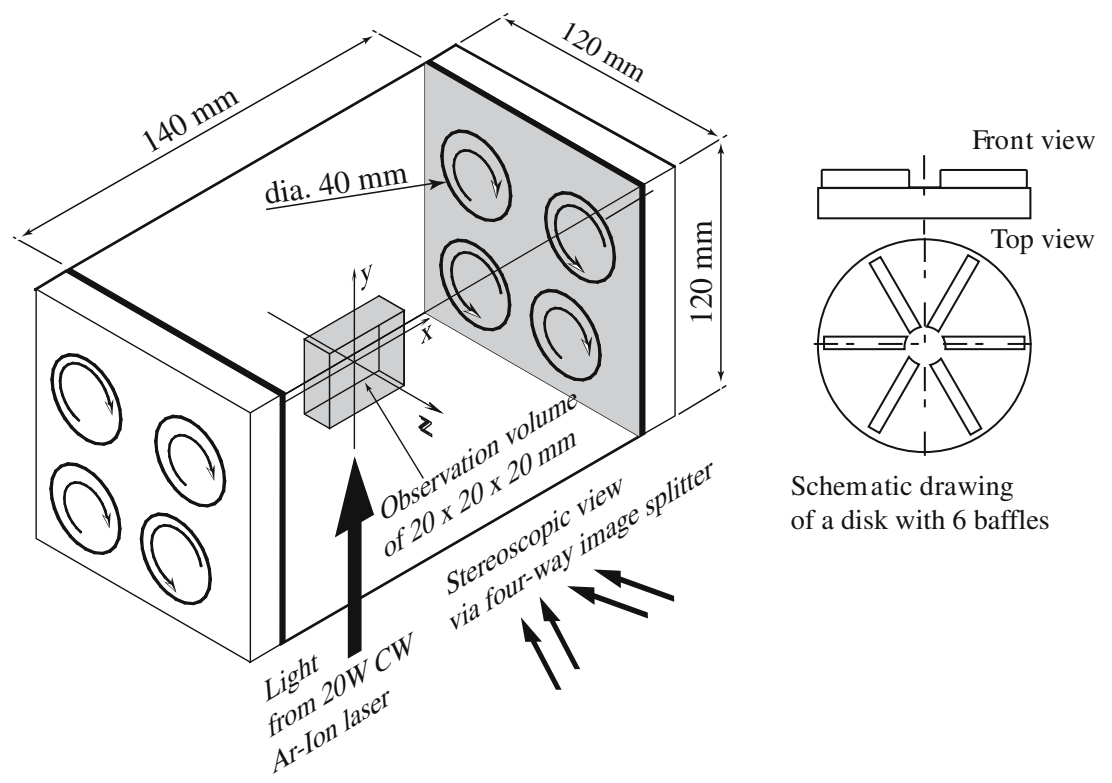

Table 1 Characteristic properties of the flow

\begin{tabular}{lllll}
\hline$\sqrt{\bar{u}^{2}}(\mathrm{~mm} / \mathrm{s})$ & $\eta(\mathrm{mm})$ & $\tau_{\eta}(\mathrm{s})$ & $\lambda(\mathrm{mm})$ & $R e_{\lambda}$ \\
\hline 10 & 0.5 & 0.25 & 5 & 50 \\
\hline
\end{tabular}

established that these particles follow water flows at our Reynolds number accurately. The observation volume was scanned using ten slices of about $3 \mathrm{~mm}$ thickness. Neighboring slices were overlapping by a ratio of about $25 \%$.

\section{Software adaptations}

\subsection{Determination of 3D particle coordinates}

The 3D PTV measurement principle is based on the synchronous acquisition of image sequences of the motion of tracer particles from four different viewing angles. After recording, the image sequences are processed to detect the particle images and extract their image coordinates, as shown by Maas (1992). With the knowledge of the previously calibrated camera position and orientation with respect to a fixed point on the wall of the flow cell, it is possible to establish corresponding particle images between the four different views and successively determine their $3 \mathrm{D}$ coordinates. This is achieved by ray-tracing through a multimedia geometry (air, glass, water; see Maas 1992 for details). With SPTV a significant increase in the yield of 3D particle coordinates per unit time step is possible, i.e. it provides a denser input of particles per unit time step for the subsequent tracking routine. To find the trajectories from the $3 \mathrm{D}$ point clouds, a tracking procedure developed by Willneff (2003), based on both image and object space information, is applied. For the presented scanning technique, some modifications of the classical PTV method both in the determination of the particle positions and in the tracking of detected particles in time are necessary.

The determination of corresponding particles in two or more images is based on the epipolar line intersection technique (Maas 1992). Stereoscopic matching is equivalent to finding the image of the same particle in the other camera views. In the strict mathematical formulation the epipolar lines intersect at corresponding particle images. The range of the illuminated region in scanning direction, which depends on the slice number ' $n$ ', limits the start and end points on the epipolar line inbetween which the corresponding particle must lie. The depth of the instantaneous observation volume, together with the tolerance band and the camera orientation, defines the search area for corresponding particles in image space. So the reduced thickness of the instantaneous observation volume reduces not only the chance of overlapping particle images, but also the number of possible candidates on the epipolar lines to match. The stereo matching thus becomes easier. For our setup, the scanned depth for one single acquisition is one order of magnitude smaller than the whole depth of the observation volume used in the classical method. The determination of corresponding particles was adopted without any modification from the classical method, apart from changing the depth of the observation volume to the slice limits as the light sheet is scanning through the volume. 
The correspondences and 3D particle positions are established sequentially, thereby filling the observation volume. Therefore, not only is the point cloud of one observation volume skewed in time, but also particles can move from one slice to the next between volume scans. Both effects need to be accounted for in the tracking and necessitate interpolation of all particle positions on a single volume time stamp. In our adapted tracking procedure we assume to find the future position of a particle in the next volume scan either in the same slice $n$ or in one of the neighboring slices at $n+1$ or $n-1$. Thus, the time interval between two particle position measurements is either $1 / f_{\mathrm{v}}$ or $1 / f_{\mathrm{v}} \pm 1 / f_{\mathrm{c}}$, respectively (see Fig. 3a). We therefore assume that we scan sufficiently fast so that a particle never moves further than one slice thickness in $x_{3}$-direction between the two volume scans. For this reason an estimate of the highest flow velocities in the flow has to be known, as will be discussed in the next subsection.

It was shown above that the effective light sheet thickness results from a dynamic part due to the sweeping motion of the sheet and a static part which results from the Gaussian beam profile across the light sheet. The resulting shape of the light intensity distribution of one slice is a "Gaussian tapered window". The dynamic width of the scan, which has been scanned by the intensity maximum, is $\Delta x_{3}, 100 \%=v_{\mathrm{s}} / f_{\mathrm{c}} \simeq 2.5 \mathrm{~mm}$, where $v_{\mathrm{s}}$ is the local scan velocity (Fig. 4). Additionally, the window is tapered on each side by half of the Gaussian shape of the laser sheet intensity profile. The main parameters governing the visibility of a particle are the particle size, viewing direction with respect to the illumination, scatter properties of the surface and/or from inside the particle and illuminating light intensity. Thus on the tapered ends of the individual slices some particles remain undetected. In this region, however, the slices overlap, so that each particle is swept over by the maximum light intensity once in either one of the frames. This contributes to the

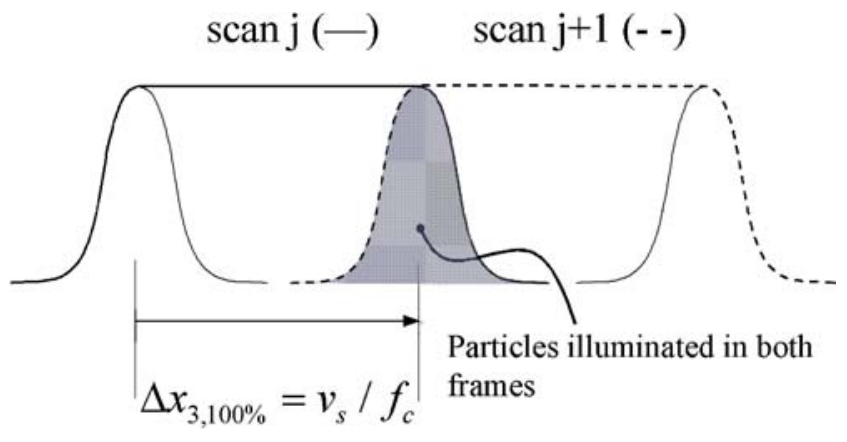

Fig. 4 Schematic illustration of the light intensity distribution through scanning with velocity $v_{\mathrm{s}}$

robustness of the method, as the probability for particles to remain undetected in the overlap region of both slices is low. Identifying double-exposed particles is straightforward, as their relative displacement for our experimental conditions is very small and lies within the order of the positioning error. To avoid that double-exposed particles are considered twice, they are identified using a nearest neighbor test with small threshold. Depending on its $x_{3}$-position within the scan, the particle that has been scanned by the intensity maximum is chosen from the pair. This intermediate identification of doubles is necessary before the data can be further processed by the tracking routines.

\subsection{Particle tracking}

The goal of the tracking procedure is to repeatedly link positions of the same particles in time from one volume scan to the next. Willneff (2003) implemented a particle tracking method based on the combined image and object space information, which led to a higher tracking efficiency for the classical PTV system. In this method,
Fig. 3 Schematic illustrations of a the time evolution versus light sheet position during four volume scans and $\mathbf{b}$ the spline interpolation scheme of a point in a trajectory

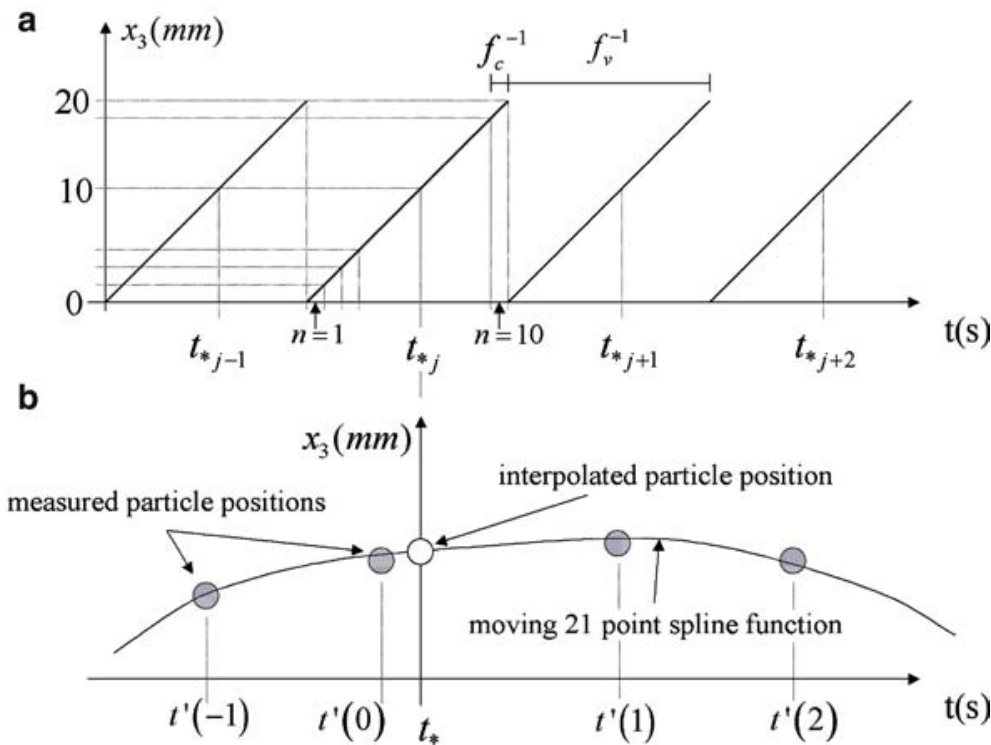


information extracted from both image and object space is used simultaneously. The same principle was implemented for the scanning technique presented. As for the classical PTV system, the tracking procedure is based on kinematic motion modeling using the following criteria for a reliable and effective assignment (see Dracos 1996): (1) the velocity of a particle is limited in all three components of the motion vector; (2) the Lagrangian acceleration of a particle is limited; and (3) in cases of ambiguities the assignment with the smallest Lagrangian acceleration is the most probable one. The first criterion defines a search volume for the positions of possible candidates, therefore an upper bound for the expected maximum velocity needs to be known (usually readily obtainable from the boundary conditions of the flow). The Lagrangian acceleration is not directly used as a threshold, but takes part of a weighting function together with the change of Lagrangian acceleration. As stated in Dracos (1996), the penalization of large acceleration magnitudes and its large changes, respectively, is a heuristic approach. However, one can expect that molecular viscosity will damp acceleration and its change in small scales. There is some evidence that this happens, for example, in the direct numerical simulation (DNS) data of Yeung and Pope (1989), who concluded that the magnitude of the Lagrangian acceleration of particles changes slowly on a time scale of approximately $0.6 T_{\mathrm{L}}$, where $T_{\mathrm{L}}$ is the integral time scale. It was verified that the chosen parameters led to stable tracking results, i.e. there is no bias towards low-intensity events.

In the classical PTV system all particles in the observation volume recorded in one frame correspond to one time step. For the scanning technique, each of the slices building up one volume scan corresponds to a different time step. The volume scan rate is set in a way that particles completely leaping one slice do not have to be considered, as the thickness of one slice is much higher than the displacement of the fastest particles between two volume scans (as said above, an upper bound for the velocity has to be known to ensure this to be true). For both the kinematic particle motion modeling and the decision criteria, no modifications were necessary to correctly track particles moving from one slice to a neighboring one, since their relative time difference is only $0.1 f_{\mathrm{v}}^{-1}$. After tracking the particles along their path across different slices, the derived trajectories consist of points separated by non-uniform time intervals. However, the velocity post-processing calls for a single time stamp for all particles of a volume scan. In the next section we describe how this is accomplished for our scanning technique.

\subsection{Trajectory processing}

Lüthi et al. (2005) presented a method that yielded filtered Lagrangian velocities and accelerations from the trajectories using a moving-spline interpolation scheme, since the velocities and especially the accelerations obtained from a simple central difference of position data showed significant noise, caused mainly by positioning errors. The interpolated positions were derived from a third-order polynomial moving-spline function fitted from an over-determined set of ten points prior and post the current point along the trajectory. The same method was modified for the presented technique not only to filter out the positioning noise, but also to interpolate particle position, velocity and acceleration in the entire observation volume at the same time stamp $t_{*}$ (defined as the time when the laser sheet passes the center of the observation volume). This way it is possible to obtain all the quantitative information of one volume scan not only filtered along trajectories, but also synchronized in time.

For clarity, the interpolation procedure for a single trajectory is presented in more detail. In SPTV, the particle positions are measured at variable time intervals depending on the position of the particle inside a volume scan. If we consider a particle $j$ in a volume scan, we denote the corresponding slice number of this particle as $n_{j}$ (as the particle can move from one slice to the next, this number can easily change several times during 21 scans). For all three position coordinates $x_{i}(i=1,2,3)$ of each point, the spline function is expanded around the time $t_{*}$ ( $t_{*}$ is set as the origin of the time axis, see Fig. 3b). Relative to $t_{*}$, the time information of each particle over the 21 trajectory points can be expressed as $t\left(j, n_{j}\right)=j / f_{\mathrm{v}}+\left(n_{j}\right.$ - $\left.\left(n_{\mathrm{s}} / 2\right)\right) / f_{\mathrm{c}}=: t^{\prime}(j)$, where $n_{\mathrm{s}}=10$ is the total number of slices. The constants $c_{i}$ are the coefficients of a third-order polynomial spline function of the form

$x_{i}(t)=c_{i, 0}+c_{i, 1} t+c_{i, 2} t^{2}+c_{i, 3} t^{3}$,

approximating the true function around the point $x_{i}\left(t_{*}\right)$, and are determined as

$c_{i}=\left(A^{\mathrm{T}} A\right)^{-1}\left(A^{\mathrm{T}} x_{i}\right)$,

where

$A=\left[\begin{array}{cccc}1 & t^{\prime}(-10) & t^{\prime 2}(-10) & t^{\prime 3}(-10) \\ 1 & t^{\prime}(-9) & t^{\prime 2}(-9) & t^{\prime 3}(-9) \\ \vdots & \vdots & \vdots & \vdots \\ 1 & t^{\prime}(10) & t^{\prime 2}(10) & t^{\prime 3}(10)\end{array}\right]$

and

$x_{i}=\left[\begin{array}{c}x_{i}\left[t^{\prime}(-10)\right] \\ x_{i}\left[t^{\prime}(-9)\right] \\ \vdots \\ x_{i}\left[t^{\prime}(10)\right]\end{array}\right]$.

Position, velocity and acceleration, $\widehat{x}_{i}\left(t_{*}\right), \widehat{u}_{i}\left(t_{*}\right), \widehat{a}_{i}\left(t_{*}\right)$, respectively, are consequently obtained as

$\widehat{x_{i}}\left(t_{*}\right)=c_{i, 0}$,

$\widehat{u_{i}}\left(t_{*}\right)=c_{i, 1}$

and

$\widehat{a}_{i}\left(t_{*}\right)=c_{i, 2}$. 
As shown in Lüthi et al. (2005), the procedure effectively acts as a low-pass filter with a cutoff frequency of about $10 \mathrm{~Hz}$, which reduces the noise originating from particle position inaccuracies. In analogy, our cutoff frequency of about $8 \mathrm{~Hz}$ is well above the maximal position signal frequency of $4 \mathrm{~Hz}$ and well below the highest resolvable frequency $25 \mathrm{~Hz}$. Now that velocities are obtained at regular time steps and time intervals with the procedure described above, spatial and temporal velocity derivatives are interpolated for every particle trajectory point as shown in Lüthi et al. (2005).

\section{Checks and results}

\subsection{Checks}

The intensity of the velocity field measured by Lüthi et al. (2005) was reaching the limit of the system capabilities with respect to trackability. Thus it was decided not to increase the spatial resolution too much and use the higher seeding for a larger field of view also. The goal was to reproduce the same flow conditions as in Lüthi et al. (2005), extend the observation volume and enhance the spatial resolution within the limits of trackability. In fact, both the observation volume and spatial resolution were nearly doubled as shown in Table 2.

For our experiment, on an average 3,900 particles per volume scan could be assigned a position in space. The linking efficiency, defined as the ratio between the number of linked and matched particles, respectively, was about $85 \%$. About 3,000 particles per volume scan could be followed over 13 time steps (equivalent to one Kolmogorov time scale $\tau_{\eta}$ ) or longer. Table 3 compares the particle numbers through the different processing steps for the classical and new techniques.

Due to the limited memory of the camera, the recorded time series is $4 \mathrm{~s}$ long. A total number of $6 \times 10^{5}$ points of the data set belong to trajectories longer than $1 \tau_{\eta}$ and $2 \times 10^{5}$ points to trajectories longer than $4 \tau_{\eta}$ (Table 4).

Table 2 Some characteristics of the PTV and SPTV experiments

\begin{tabular}{lll}
\hline & $\begin{array}{l}\text { Observed } \\
\text { volume }\left(\mathrm{cm}^{3}\right)\end{array}$ & $\begin{array}{l}\text { Inter-particle } \\
\text { distance }(\mathrm{mm})\end{array}$ \\
\hline PTV & 4.5 & 2.3 \\
SPTV & 8 & 1.2 \\
\hline
\end{tabular}

Table 3 Comparison of particle numbers for the PTV and SPTV experiments along the chain of processing steps

\begin{tabular}{llll}
\hline & $\begin{array}{l}\text { No. of } \\
\text { particles }\end{array}$ & $\begin{array}{l}\text { No. of } \\
\text { correspondences }\end{array}$ & $\begin{array}{l}\text { No. of } \\
\text { links }\end{array}$ \\
\hline PTV & 1,500 & 800 & 600 \\
SPTV & 5,000 & 3,900 & 3,500 \\
\hline
\end{tabular}

Table 4 Characteristics of the statistical sets of the PTV and SPTV experiments

\begin{tabular}{lllll}
\hline & $\begin{array}{l}\text { Recording } \\
\text { time }(\mathrm{s})\end{array}$ & $\tau_{\eta}(\mathrm{s})$ & $\begin{array}{l}\text { Frames } / \tau_{\eta} \\
(1 / \mathrm{s})\end{array}$ & $\begin{array}{l}\text { Recording } \\
\text { time } / \tau_{\eta}\end{array}$ \\
\hline PTV & 100 & 0.23 & 14 & $\begin{array}{l}435 \\
16\end{array}$ \\
SPTV & 4 & 0.25 & 13 & \\
\hline
\end{tabular}

Checks based on precise kinematical relations are presented to validate the modifications of the introduced technique and to assess the quality of the data itself. Due to the incompressibility of water ideally the trace of $\left(\left(\partial u_{i}\right) /\left(\partial x_{j}\right)\right)$ should be zero, i.e.

$-\frac{\partial u_{i}}{\partial x_{i}}=\frac{\partial u_{j}}{\partial x_{j}}+\frac{\partial u_{k}}{\partial x_{k}}$.

Figure 5 shows the joint PDFs of $-\left(\partial u_{i} / \partial x_{i}\right)$ versus $\left(\partial u_{j} / \partial x_{j}\right)+\left(\partial u_{k} / \partial x_{k}\right)$ (no summation over $i, j, k$ is applied). The aspect ratios of the contour surfaces are significantly smaller than the ones shown in Lüthi et al. (2005) (the values are compared in Table 5). This is a first indication that the lower inter-particle distance (and thus higher spatial resolution) leads to a higher quality of velocity derivatives.

A more strict check involves several quantities, each of which are derived in a different manner, the Lagrangian accelerations $a_{i}=\mathrm{D} u_{i} / \mathrm{D} t$, local accelerations $a_{1}, i=\partial u_{i} / \partial t$ and convective accelerations $a_{\mathrm{c}}, i=u_{j} \partial u_{i} / \partial x_{j}$. These quantities are related by the following equation:

$\frac{\mathrm{D} u_{i}}{\mathrm{D} t}=\frac{\partial u_{i}}{\partial t}+u_{j} \frac{\partial u_{i}}{\partial x_{j}}$

The left-hand side of the equation is derived from the trajectory of a single particle, whereas the right-hand terms need common information from neighboring particles. In a spatially under-resolved low-pass-filtered flow field, the divergence check might still be satisfactory with underestimated velocity gradients. The acceleration check provides a better assessment of the accuracy of velocity derivatives, because $a_{i}$, compared to the other two quantities, is obtained in a more straightforward manner. Joint PDFs of $a_{i}$ versus $a_{1}, i+a_{\mathrm{c}}, i$ are shown in Fig. 6. Similar to the divergence check, the aspect ratios of the contour surfaces are lower than the ones obtained applying the classical PTV system, suggesting again that the quality of the data has improved (the values are shown in Table 5). The higher aspect ratio for the third acceleration component is associated with the $x_{3}$-component inaccuracy as explained by Lüthi et al. (2005). Due to the arrangement of the four view directions with respect to the observation volume, the $x_{3}$-position signal is less accurate than $x_{1}$ and $x_{2}$.

The relative divergence, $\delta$, defined as

$\delta=\frac{\left\|\frac{\partial u_{1}}{\partial x_{1}}+\frac{\partial u_{2}}{\partial x_{2}}+\frac{\partial u_{3}}{\partial x_{3}}\right\|}{\left\|\frac{\partial u_{1}}{\partial x_{1}}\right\|+\left\|\frac{\partial u_{1}}{\partial x_{2}}\right\|+\left\|\frac{\partial u_{3}}{\partial x_{3}}\right\|}$ 
Fig. 5 Joint PDFs of $-\left(\partial u_{i} / \partial x_{i}\right)$ versus $\left(\partial u_{j} / \partial x_{j}\right)+\left(\partial u_{k} / \partial x_{k}\right)$ (here, no summation over $i, j, k$ is applied)
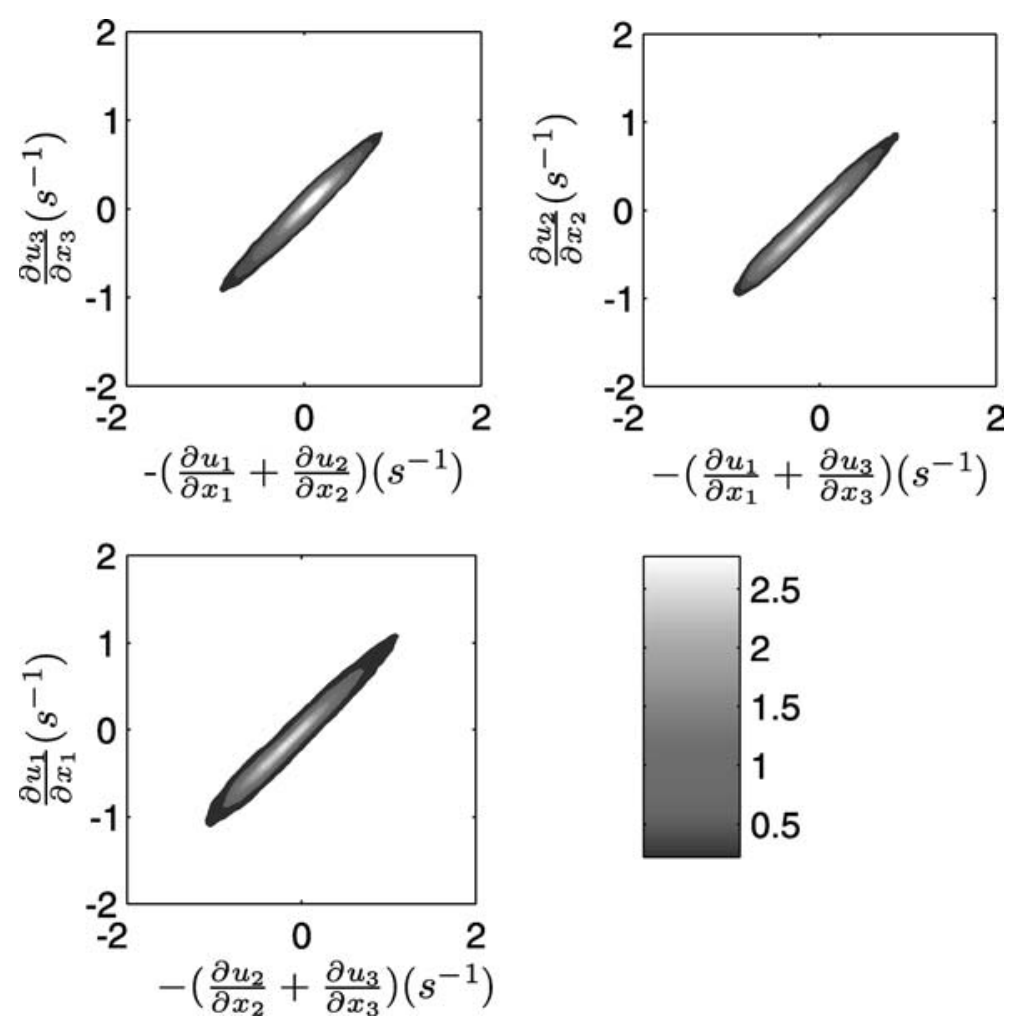

Table 5 Aspect ratios of the contour surfaces obtained from the "divergence check" and "acceleration check" compared for the PTV and SPTV experiments

Divergence check

Acceleration check

\begin{tabular}{lll}
\hline PTV & $1 / 5$ & $1 / 3.5$ \\
SPTV & $1 / 10$ & $1 / 4.5$ \\
\hline
\end{tabular}

is used for a weighted fit along particle trajectories as a measure for the local interpolation quality (Lüthi et al. 2005 ). For the experiment presented, $67 \%$ of the data points are of high quality, i.e. $\delta \leq 0.1$. Therefore, the whole data set is more reliable and the effect of the weighted polynomial is reduced. This effect is also visible in the last check presented. Figure 7 shows the mean relative divergence, $\langle\delta\rangle$, as defined in expression 12, plotted
Fig. 6 The expression (D $u_{i} / \mathrm{D}$ $t)=\left(\partial u_{i} / \partial t\right)+u_{j}\left(\partial u_{i} / \partial x_{j}\right)$ is checked for each component $i$, with joint PDFs of $a_{1}, i+a_{\mathrm{c}}, i$ versus $a_{i}$
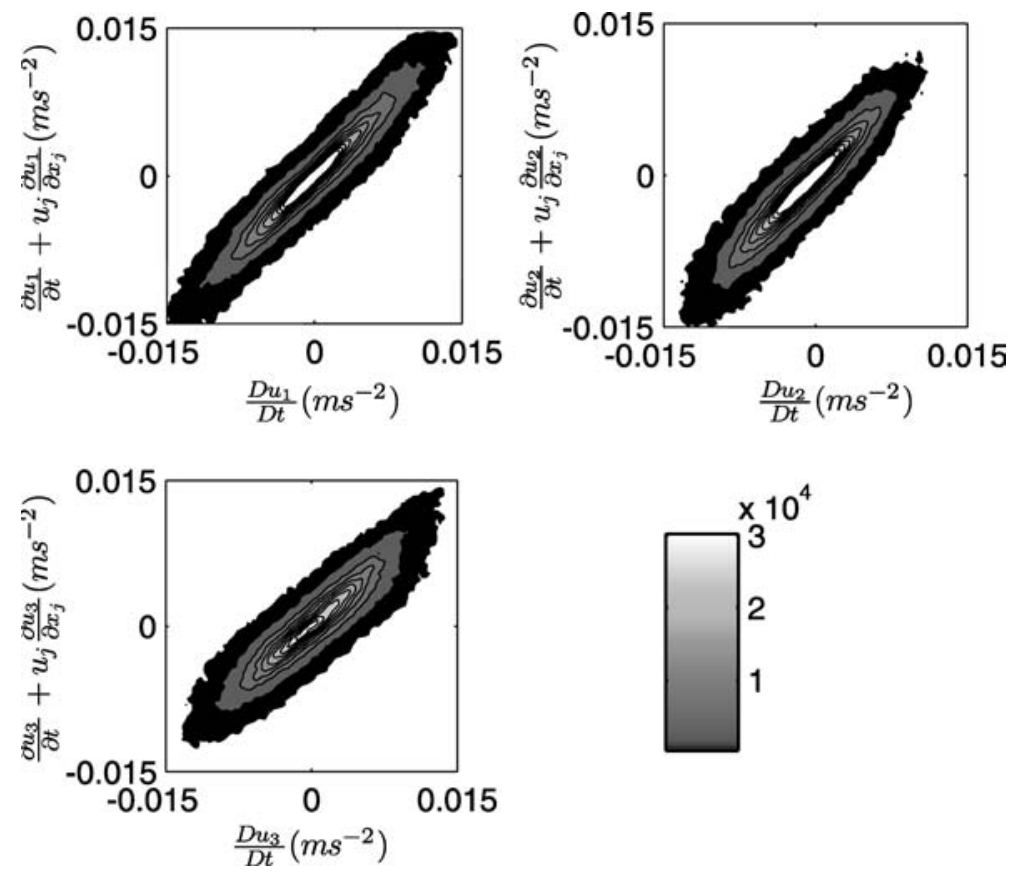


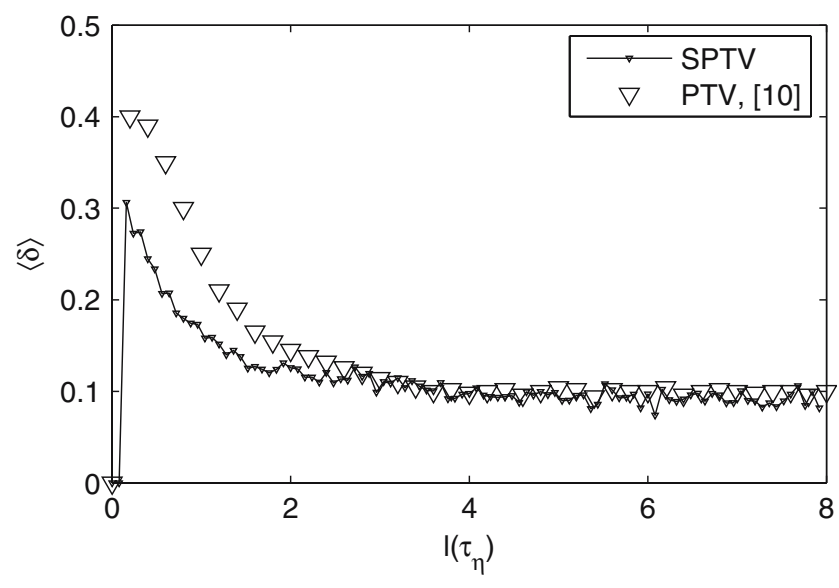

Fig. 7 Relative divergence $\langle\delta\rangle$ plotted over trajectory length $l$

as a function of the trajectory length $l$. Compared to the plot shown in Lüthi et al. (2005), $\langle\delta\rangle$, after a lower peak, starts to decay earlier towards a common value of 0.1 .

The above checks show not only that velocity derivatives can be obtained correctly with the presented technique, but also that their quality is significantly improved compared to the classical PTV method. Following the approach shown in Lüthi et al. (2005) (Sect. 2.4.1), the accuracy analysis was repeated for SPTV, using the aspect ratios shown in Table 5. With a measured $\operatorname{rms}(\partial u / \partial x)$ of $1.5 \mathrm{~s}^{-1}$ the accuracy of $(\partial u / \partial x)$ relative to its rms, $\epsilon_{\partial u / \partial x}$, is approximately $7 \%$. Thus, compared to the experiment in Lüthi et al. (2005), the accuracy of velocity derivatives is improved by a factor 2. Further, with a measured $\operatorname{rms}\left(a_{\mathrm{c}}, i\right) \sim 13 \mathrm{~mm} / \mathrm{s}^{2}$, the accuracy for the convective acceleration (assumed to be of equal order than the local acceleration) relative to its rms gives $15 \%$, while Lüthi et al. (2005) estimated an accuracy of $20 \%$ for this quantity.

\subsection{Results}

The statistics shown in this section are derived from data points stemming from particle trajectories longer than 13 frames, with a relative divergence, $\delta$, equal or smaller than 0.1 . The number of data points that satisfy this criterion are $3.8 \times 10^{5}$.

Some selected results on the statistics of vorticity and strain are briefly presented in this section, for the sake of comparison between the two methods. First, the PDFs of enstrophy and strain production, $\omega_{i} \omega_{j} s_{i j}$ and $-s_{i j} s_{j k} s_{k i}$, normalized with their respective mean values are shown in Fig. 8. We compare the results obtained by SPTV to the results of Liberzon et al. (2005), who used the classical PTV of Lüthi et al. (2005) in the same experimental facility and with the same experimental parameters. The agreement between the results in Fig. 8 is satisfactory, where PDFs of the production terms show clearly positively skewed distributions, as shown also in Lüthi et al. (2005) and Su and Dahm (1996b).

Second, we take a look at the PDFs of the eigenvalues $\Lambda_{1,2,3}$ of the rate of strain tensor, $s_{i j}$. Figure 9 shows a fundamental property of turbulence, i.e. the positive skewness of $\Lambda_{2}$. The magnitude of $\left\langle\Lambda_{2}\right\rangle \approx$ $0.48 \mathrm{~s}^{-1}$ is small compared to $\left\langle\Lambda_{1}\right\rangle \approx 2.4 \mathrm{~s}^{-1}$ and $\left\langle\Lambda_{3}\right\rangle \approx$ $-2.9 \mathrm{~s}^{-1}$. The ratio of $\left\langle\Lambda_{1}\right\rangle:\left\langle\Lambda_{2}\right\rangle:\left\langle\Lambda_{3}\right\rangle$ is consistent with Liberzon et al. (2005) and Lüthi et al. (2005) and references therein.

Figure 10 shows the PDFs of $\left|\cos \left(\omega, \lambda_{i}\right)\right|$ conditioned on weak and strong strains, illustrating the orientation of vorticity $\omega$, relative to the eigenframe of the rate of strain tensor $s_{i j}$. For strong events a predominant
Fig. 8 PDFs of $\omega_{i} \omega_{j} s_{i j}$ and $-s_{i j} s_{j k} s_{k i}$ normalized with their mean values $\left\langle\omega_{i} \omega_{j} s_{i j}\right\rangle$ and $\left\langle-(4 / 3) s_{i j} s_{j k} s_{k i}\right\rangle$, respectively, as obtained by SPTV and the PTV experiments of Liberzon et al. (2005)

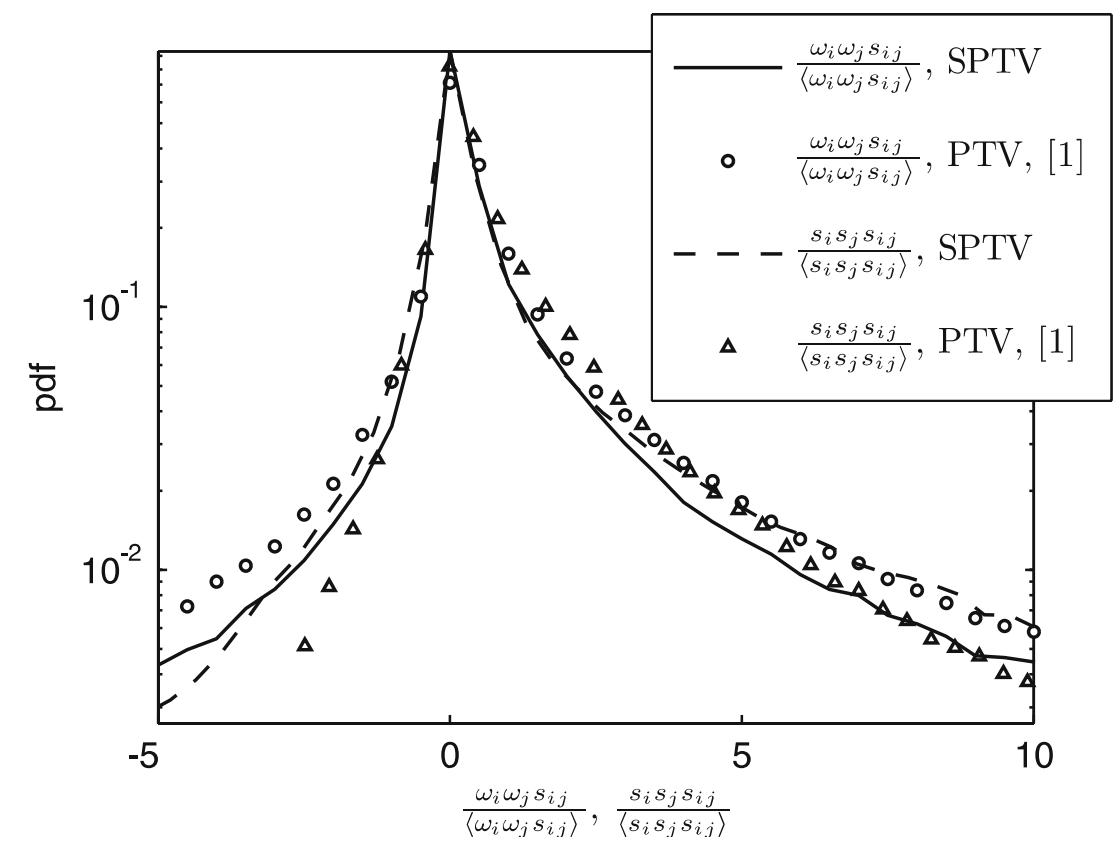




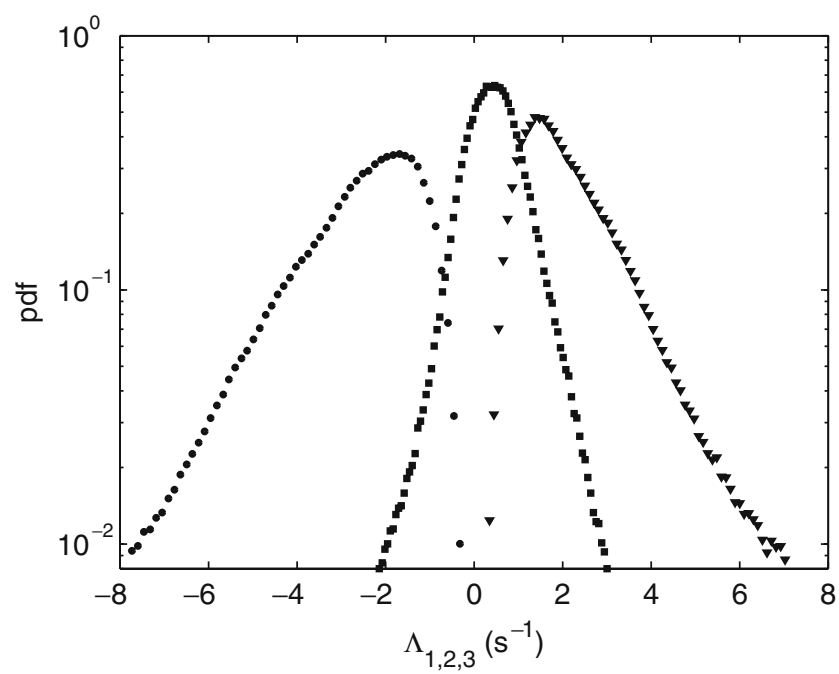

Fig. 9 PDFs of the eigenvalues, $\Lambda_{1,2,3}$, of the rate of strain tensor $s_{i j}$. Inverted triangle $\Lambda_{1}$, square $\Lambda_{2}$, circle $\Lambda_{3}$

alignment of $\omega$ with the intermediate eigenvector $\lambda_{2}$ of the rate of strain tensor persists (see also Lüthi et al. 2005). As stated in Tsinober (2001), this result is one of the intrinsic properties of turbulent flows.

Last, Fig. 11 shows a joint PDF plot of vortex stretching versus $(1 / 2) \mathrm{D} \omega^{2} / \mathrm{D} t$. For a flow with a statistically stationary level of enstrophy, it is clear that enstrophy production is balanced by its viscous destruction (Tsinober 2001) as

$\left\langle\omega_{i} \omega_{j} s_{i j}\right\rangle=-\left\langle v \omega_{i} \nabla^{2} \omega_{i}\right\rangle$.

As already pointed out by Lüthi et al. (2005), the variations of $(1 / 2) \mathrm{D} \omega^{2} / \mathrm{D} t$ are much larger than those

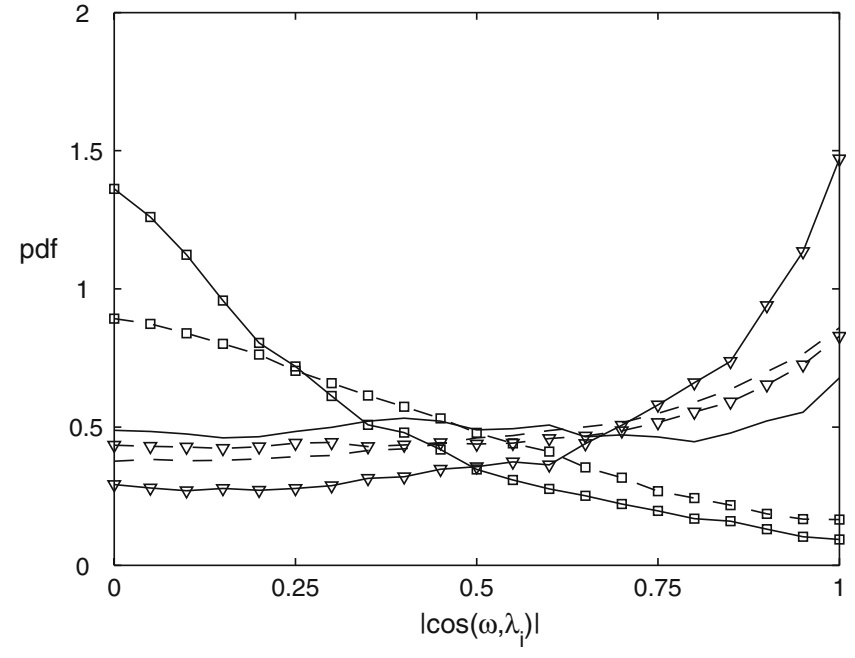

Fig. 10 PDFs of $\cos \left(\omega, \lambda_{i}\right)$ conditioned on weak and strong $s^{2}$. Dotted line $\cos \left(\omega, \lambda_{1}\right) s^{2}<\left\langle s^{2}\right\rangle$, full line $\cos \left(\omega, \lambda_{1}\right) s^{2}>\left\langle s^{2}\right\rangle$, inverted triangle with dotted line $\cos \left(\omega, \lambda_{2}\right) s^{2}<\left\langle s^{2}\right\rangle$, inverted triangle with full line $\left.\cos \left(\omega, \lambda_{2}\right) s^{2}\right\rangle\left\langle s^{2}\right\rangle$, square with dotted line $\cos \left(\omega, \lambda_{3}\right) s^{2}<\left\langle s^{2}\right\rangle$, square with full line $\left.\cos \left(\omega, \lambda_{3}\right) s^{2}\right\rangle\left\langle s^{2}\right\rangle$

for vortex stretching. Apparently there is no simple relation between production and change of magnitude of enstrophy. The action of the viscous term $v$ $\omega_{i} \nabla^{2} \omega_{i}$ in balancing overall enstrophy production must be strongly non-local in space, since (see Tsinober 2001)

$\frac{\mathrm{D}\left(\frac{1}{V} \int_{V} \omega^{2} \mathrm{~d} V\right)}{\mathrm{D} t} \ll \frac{1}{V} \int_{V} \omega_{i} \omega_{j} s_{i j} \mathrm{~d} V \sim-\frac{v}{V} \int_{V} \omega_{i} \nabla^{2} \omega_{i} \mathrm{~d} V$.

Fig. 11 Joint PDF plot of vorticity production versus change of enstrophy, both axes normalized with $\left\langle\omega_{i} \omega_{j} s_{i j}\right\rangle$

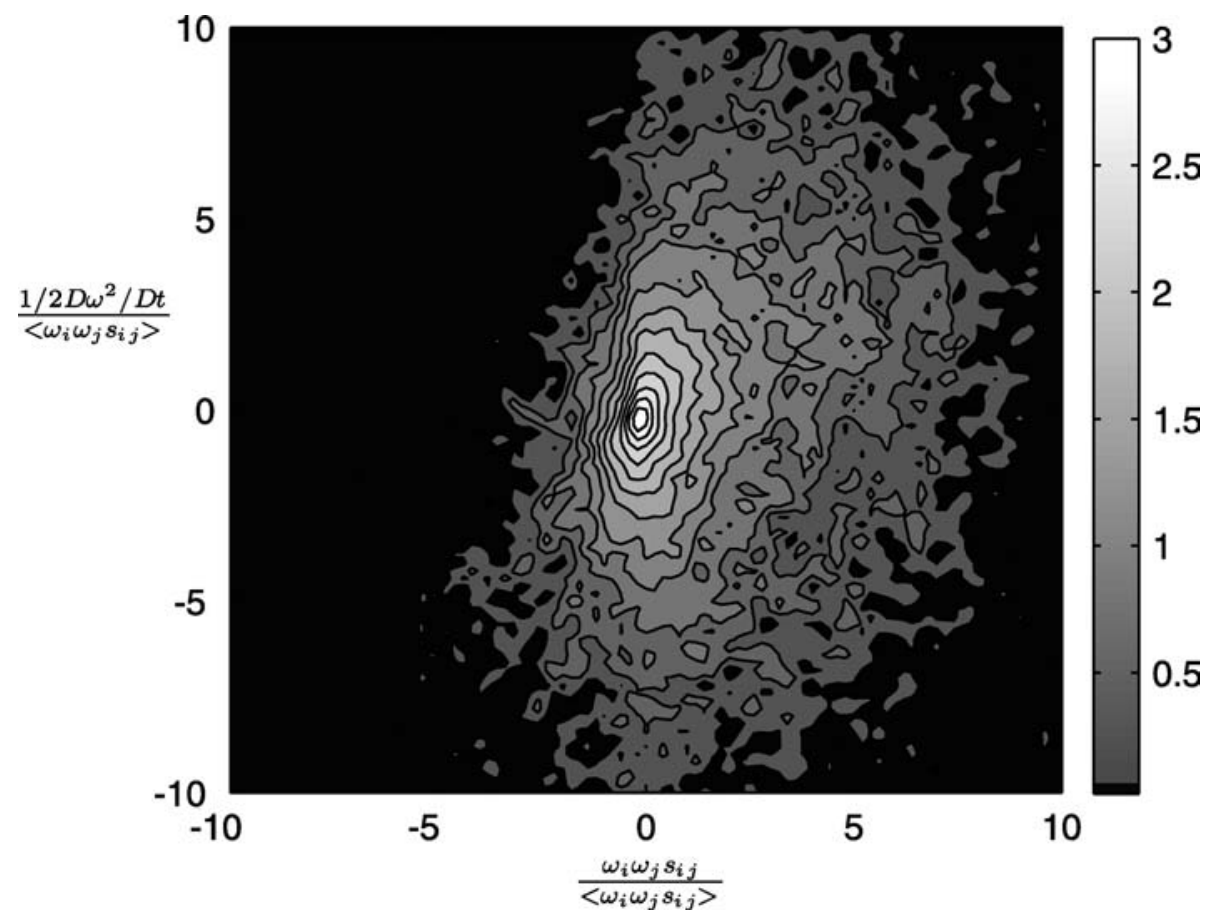




\section{Conclusions}

In this article, a scanning technique for PTV (SPTV) is presented. So far, SPTV yielded 3,500 velocity vectors per unit time step, using just one single high-speed camera. Compared to classical PTV, both the observation volume and the spatial resolution were nearly doubled. Limited memory of the camera allowed a recording time of $4 \mathrm{~s}$ only, thus posing limitations on the convergence of some statistics. However, hardware improvements can easily overcome the mentioned limitation. SPTV offers enhanced data quality compared to the classical PTV and represents an excellent tool for turbulence investigation. In particular, SPTV has the potential to provide a spatial resolution high enough for attempting an investigation on the role played by the viscous term in the enstrophy balance.

Acknowledgements This work was supported by ETH Grant No. 0-20151-03.

\section{References}

Brücker Ch (1996a) 3-D scanning-particle-image-velocimetry: technique and application to a spherical cap wake flow. Appl Sci Res 56(2-3):157-179

Brücker Ch (1996b) 3-D PIV via spatial correlation in a colorcoded light-sheet. Exp Fluids 21:312-314

Brücker Ch (1997) 3-D scanning PIV applied to a air flow in a motored engine using digital high speed video. Meas Sci Technol 8:1480-1492

Chang T, Tatterson G (1983) Application of image processing to the analysis of three-dimensional flow fields. Opt Eng 23:283-287

Dracos Th (ed) (1996) Three-dimensional velocity and vorticity measuring and image analysis techniques. Dordrecht, Kluwer, Dordrecht, ISBN 0-7923-4256-9

Guezennec YG, Zhao Y, Gieseke T (1994) High speed 3-D scanning particle image velocimetry (3-D SPIV). Developments in laser techniques and applications to fluid mechanics. In: Adrian RJ et al (eds) Proceedings of the 7th international symposium, Lisbon, Portugal, pp 11-14
Hori T, Sakakibara J (2004) High-speed scanning stereoscopic PIV for 3D vorticity measurement in liquids. Meas Sci Technol 15:1067-1078

Kasagi N, Nishino K (1991) Probing turbulence with threedimensional particle tracking velocimetry. Exp Therm Fluid Sci 4:601-612

Liberzon A, Guala M, Lüthi B, Kinzelbach W, Tsinober A (2005) Turbulence in dilute polymer solutions. Phys Fluids 17:031707

Lüthi B, Tsinober A, Kinzelbach W (2005) Lagrangian measurement of vorticity dynamics in turbulent flow. J Fluid Mech 528:87-118

Maas H-G (1992) Digitale Photogrammetrie in der dreidimensionalen Strömungsmesstechnik. Dissertation (ETH Zürich)

Maas H-G, Gruen D, Papantoniou D (1993) Particle tracking velocimetry in three-dimensional flows. Exp Fluids 15:133146

Merkel GJ, Rys F, Rys P, Dracos Th (1998) Laser induced fluorescence tomography in passive and reactive scaler fields. Physical processes and chemical reactions in liquid flows. IAHR Monograph Series, pp 57-68

Nishino K, Kasagi N, Hirata M (1988) Three-dimensional particle tracking velocimetry based on automated image processing. ASME J Fluids Eng 111(4):384-391

Papantoniou D, Maas H-G (1990) Recent advances in 3-D particle tracking velocimetry. In: Proceedings of the 5th international symposium on the application of laser techniques in fluid mechanics, Lisbon, July 9-12

Racca R, Dewey J (1988) A method for automatic particle tracking in a three-dimensional flow field. Exp Fluids 6:25-32

Sakakibara J, Adrian RJ (1999) Whole field measurement of temperature in water using a two-color laser induced fluorescence. Exp Fluids 26:7-15

Su L, Dahm W (1996a) Scalar imaging velocimetry measurements of the velocity gradient tensor field in turbulent flows. I. Assessment of errors. Phys Fluids 8(7):1869-1882

Su L, Dahm W (1996b) Scalar imaging velocimetry measurements of the velocity gradient tensor field in turbulent flows. II. Experimental Results. Phys Fluids 8(7):1883-1906

Tsinober A (2001) An informal introduction to turbulence. Kluwer, Dordrecht, ISBN: $140200110 \mathrm{X}$

Willneff J (2003) A spatio-temporal matching algorithm for 3D particle tracking velocimetry. Dissertation (ETH Zürich)

Yeung PK, Pope SB (1989) Lagrangian statistics from direct numerical simulations of isotropic turbulence. JFM 207:531586 